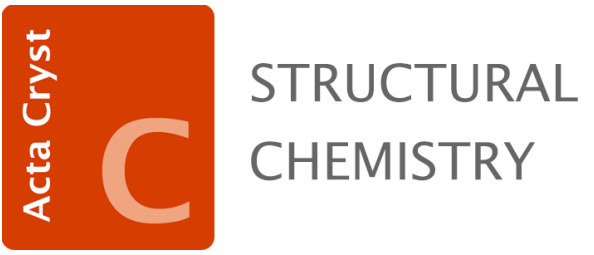

Volume 74 (2018)

Supporting information for article:

A novel platinum-based anticancer drug: a complete vibrational study A.L.M. Batista de Carvalho, S.F. Parker, L.A.E Batista de Carvalho and M.P.M. Marques 


\title{
Novel Platinum-based Anticancer Drug: a Complete Vibrational Study
}

\author{
A.L.M. Batista de Carvalho ${ }^{a}$, S.F. Parker ${ }^{b}$, L.A.E Batista de Carvalho ${ }^{\text {a* }}$ and M.P.M. Marques ${ }^{\text {ac }}$ \\ aUnidade I\&D Química-Física Molecular, Department of Chemistry, University of Coimbra, \\ Coimbra, 3004-535, Portugal \\ ' ISIS Facility, Rutherford Appleton Laboratory, OX11 0QX, England \\ 'Department of Life Sciences, University of Coimbra, 3001-401, Portugal
}

\section{Supplementary material}


Cartesian coordinates for the solid phase conformer of $\mathrm{Pt}_{2} \mathrm{Spm}$

\begin{tabular}{cccc} 
Symbol & $\mathrm{X}$ & $\mathrm{Y}$ & $\mathrm{Z}$ \\
$\mathrm{Pt}$ & -4.0763460 & 0.2599500 & -0.0711900 \\
$\mathrm{~N}$ & -4.7042880 & -1.2505510 & 1.1952630 \\
$\mathrm{H}$ & -4.0093990 & -1.4348830 & 1.9180640 \\
$\mathrm{H}$ & -5.5090750 & -0.8305460 & 1.6703750 \\
$\mathrm{~N}$ & -2.7753210 & -0.9997980 & -1.1000870 \\
$\mathrm{H}$ & -2.6311460 & -0.4583050 & -1.9576880 \\
$\mathrm{Cl}$ & -3.4313100 & 1.9034930 & -1.5997620 \\
$\mathrm{Cl}$ & -5.5777470 & 1.6029530 & 1.1057380 \\
$\mathrm{C}$ & -3.4288040 & -2.2757080 & -1.4706430 \\
$\mathrm{C}$ & -5.0813830 & -2.5005400 & 0.5058540 \\
$\mathrm{C}$ & -3.9225200 & -3.0978560 & -0.2828850 \\
$\mathrm{H}$ & -4.2696920 & -2.0217360 & -2.1215660 \\
$\mathrm{H}$ & -2.7201180 & -2.8800590 & -2.0517570 \\
$\mathrm{H}$ & -5.4389310 & -3.2317580 & 1.2402950 \\
$\mathrm{H}$ & -5.9122090 & -2.2550230 & -0.1599160 \\
$\mathrm{H}$ & -4.2600960 & -4.0612370 & -0.6796620 \\
$\mathrm{H}$ & -3.0925760 & -3.3361670 & 0.3934570 \\
$\mathrm{C}$ & -1.4451770 & -1.1904430 & -0.4706190 \\
$\mathrm{C}$ & -0.6797340 & 0.1152420 & -0.3269660 \\
$\mathrm{H}$ & -0.8714360 & -1.9045040 & -1.0775940 \\
$\mathrm{H}$ & -1.5954660 & -1.6450900 & 0.5119810 \\
$\mathrm{H}$ & -0.5526630 & 0.5724940 & -1.3155330 \\
$\mathrm{H}$ & -1.2823070 & 0.8198300 & 0.2551660 \\
$\mathrm{C}$ & 0.6797340 & -0.1152420 & 0.3269660 \\
$\mathrm{C}$ & 1.4451770 & 1.1904430 & 0.4706190 \\
$\mathrm{H}$ & 0.5526630 & -0.5724940 & 1.3155330 \\
$\mathrm{H}$ & 1.2823070 & -0.8198300 & -0.2551660 \\
$\mathrm{~N}$ & 2.7753210 & 0.9997980 & 1.1000870 \\
$\mathrm{H}$ & 0.8714360 & 1.9045040 & 1.0775940 \\
$\mathrm{H}$ & 1.5954660 & 1.6450900 & -0.5119810 \\
$\mathrm{Pt}$ & 4.0763460 & -0.2599500 & 0.0711900 \\
$\mathrm{H}$ & 2.6311460 & 0.4583050 & 1.9576880 \\
$\mathrm{H}$ & 3.4288040 & 2.2757080 & 1.4706430 \\
$\mathrm{H}$ & 5.9122090 & 2.2550230 & 0.1599160 \\
$\mathrm{H}$ & 4.7042880 & 1.2505510 & -1.1952630 \\
$\mathrm{Cl}$ & 3.4313100 & -1.9034930 & 1.5997620 \\
$\mathrm{Cl}$ & 5.5777470 & -1.6029530 & -1.1057380 \\
$\mathrm{C}$ & 3.9225200 & 3.0978560 & 0.2828850 \\
$\mathrm{H}$ & 4.2696920 & 2.0217360 & 2.1215660 \\
$\mathrm{H}$ & 2.7201180 & 2.8800590 & 2.0517570 \\
$\mathrm{H}$ & 4.0093990 & 1.4348830 & -1.9180640 \\
$\mathrm{H}$ & 5.5090750 & 0.8305460 & -1.6703750 \\
$\mathrm{H}$ & 5.0813830 & 2.5005400 & -0.5058540 \\
$\mathrm{H}$ & 4.2600960 & 4.0612370 & 0.6796620 \\
\hline & 3.0925760 & 3.3361670 & -0.3934570 \\
$\mathrm{H}$ & & & -1.2402950 \\
$\mathrm{H}$ & 5.4389310 & 3.2317580 & \\
$\mathrm{H}$ & & &
\end{tabular}


Cartesian coordinates for the most stable conformer of $\mathrm{Pt}_{2} \mathrm{Spm}$

$\begin{array}{cccc}\text { Symbol } & \mathrm{X} & \mathrm{Y} & \mathrm{Z} \\ \mathrm{Pt} & 2.0794570 & -0.1879770 & -0.3867160 \\ \mathrm{~N} & 1.3683240 & 1.5014850 & -1.3613840 \\ \mathrm{H} & 1.8850180 & 1.4881060 & -2.2403040 \\ \mathrm{H} & 0.3855230 & 1.3430200 & -1.6259360 \\ \mathrm{~N} & 2.6970670 & 0.7642130 & 1.3881900 \\ \mathrm{H} & 3.6983290 & 0.5928940 & 1.2828990 \\ \mathrm{Cl} & 2.9606830 & -2.1816900 & 0.4893960 \\ \mathrm{Cl} & 1.5216840 & -1.2696900 & -2.3928160 \\ \mathrm{C} & 2.5206920 & 2.2333740 & 1.4790990 \\ \mathrm{C} & 1.4616570 & 2.8370200 & -0.7413440 \\ \mathrm{C} & 2.6768810 & 2.9764920 & 0.1618530 \\ \mathrm{H} & 1.5204990 & 2.4242980 & 1.8725050 \\ \mathrm{H} & 3.2389480 & 2.6102710 & 2.2179260 \\ \mathrm{H} & 1.4826190 & 3.5915390 & -1.5336910 \\ \mathrm{H} & 0.5424800 & 2.9947500 & -0.1707350 \\ \mathrm{H} & 2.8037260 & 4.0382000 & 0.3994500 \\ \mathrm{H} & 3.5884870 & 2.6625780 & -0.3617040 \\ \mathrm{C} & 2.3424520 & 0.1117510 & 2.6898760 \\ \mathrm{C} & 0.8664660 & 0.1155120 & 3.0651970 \\ \mathrm{H} & 2.9103640 & 0.6364570 & 3.4696270 \\ \mathrm{H} & 2.7125110 & -0.9111970 & 2.6229820 \\ \mathrm{H} & 0.4708510 & 1.1378540 & 3.0873510 \\ \mathrm{H} & 0.8231910 & -0.2357440 & 4.1041780 \\ \mathrm{C} & -0.0318940 & -0.7528930 & 2.1885100 \\ \mathrm{C} & -1.4896040 & -0.6029220 & 2.5924340 \\ \mathrm{H} & 0.0695830 & -0.4322930 & 1.1445630 \\ \mathrm{H} & 0.3072570 & -1.7927530 & 2.2290500 \\ \mathrm{~N} & -2.4755470 & -0.9159090 & 1.5159440 \\ \mathrm{H} & -1.6904020 & 0.4397780 & 2.8468300 \\ \mathrm{H} & -1.7328550 & -1.2217190 & 3.4668340 \\ \mathrm{Pt} & -2.1988460 & 0.2508730 & -0.1964850 \\ \mathrm{H} & -3.3535600 & -0.5179690 & 1.8524070 \\ \mathrm{H} & -3.1106080 & -2.7380530 & -1.3725350 \\ \mathrm{C} & -2.7152600 & -2.3630950 & 1.2989690 \\ \mathrm{H} & -1.6082640 & -1.2882240 & -1.4404970 \\ \mathrm{H} & -2.8933290 & 2.0476650 & 1.1480420 \\ \mathrm{H} & -1.9386770 & -1.0082420 & -2.3651700 \\ \mathrm{H} & -2.0429490 & -2.6637050 & -1.1448160 \\ \mathrm{H} & -1.8737460 & 1.6046340 & -2.0884010 \\ \mathrm{H} & -1.7835140 & -3.0437000 & 0.3037220 \\ \mathrm{H} & -2.7463370 & -2.4612560 & 0.9446590 \\ \mathrm{H} & -0.7316760 & -4.1228690 & 0.4000730 \\ \mathrm{H} & -2.8699020 & 0.5510950 \\ \mathrm{H} & & & \\ \mathrm{H} & -2.8671370 & 2.2708860 \\ \mathrm{H} & & \end{array}$

